\title{
MOTIVAÇÃO À PRÁTICA REGULAR DE ATIVIDADES FÍSICAS: UM ESTUDO COM PRATICANTES DE TAEKWONDO
}

\section{Lisiane Borges Rocha Sampedro}

Universidade Federal do Rio Grande do Sul, Porto Alegre, Rio Grande do Sul, Brasil

\section{Juliana Moraes Rocha}

Universidade Federal do Rio Grande do Sul, Porto Alegre, Rio Grande do Sul, Brasil

\section{Roberto Tierling Klering}

Universidade Federal do Rio Grande do Sul, Porto Alegre, Rio Grande do Sul, Brasil

\section{Ricardo Pedrozo Saldanha}

Universidade Federal do Rio Grande do Sul, Porto Alegre, Rio Grande do Sul, Brasil

\section{Marcos Alencar Abaide Balbinotti}

Université du Québec à Trois-Rivières, Canadá

\section{Carlos Adelar Abaide Balbinotti}

Universidade Federal do Rio Grande do Sul, Porto Alegre, Rio Grande do Sul, Brasil

\section{Resumo}

O objetivo desse estudo é investigar, dentre as principais dimensões motivacionais (Controle de Estresse, Saúde, Sociabilidade, Competitividade, Estética e Prazer), o que mais motiva à prática regular do Taekwondo. $\mathrm{O}$ instrumento utilizado para a coleta de dados foi o Inventário de Motivação à Prática Regular de Atividades Físicas e/ou Esportivas - IMPRAFE-54. A amostra foi composta por 50 praticantes de Taekwondo da faixa etária de 12 a 51 anos, de ambos os sexos. Entre os resultados observou-se que a dimensões motivacionais Saúde e Prazer estatisticamente indissociáveis $(\mathrm{p}<0,05)$ são as que mais motivam os praticantes de Taekwondo.

Palavras-chave: Motivação. Atividade Motora. Esportes.

\section{Introdução}

$\mathrm{O}$ motivos que estão associados à prática regular de atividades físicas e esportivas podem ser vários. Após a realização de alguns estudos (BALBINOTTI; SILVA, 2003; BALBINOTTI, 2004, 2010; 
BALBINOTTI; BARBOSA, 2006, 2008; BALBINOTTI; SALDANHA; BALBINOTTI, 2009; BALBINOTTI; CAPOZZOLI, 2008; CAPAZZOLI, 2006; SALDANHA et al. 2008; FONTANA, 2010 LEITE GONÇALVES; BALBINOTTI, 2011), Balbinotti (2010) constatou que os principais motivos que levam à prática regular de atividades físicas e/ou esportivas são os seguintes: Controle de estresse, Saúde, Sociabilidade, Competitividade, Estética e Prazer.

O Controle de estresse diz respeito à prática de atividade física com o objetivo de reduzir os níveis de estresse gerado na vida diária (McDONALD; HODGOON, 1991). O motivo Saúde pode ser compreendido de diversas formas, desde a ausência de doenças até a promoção da vida e do bem-estar, em um sentido que integre os aspectos biológicos, psicológicos e sociais. A Sociabilidade, por sua vez, se refere à tendência natural da maioria dos seres humanos de buscar agrupar-se socialmente, de suprir a necessidade de vínculo, de pertencimento, de aceitação e de identificação (SILVA, 2000). A Competitividade pode ser considerada uma razão forte para o engajamento do indivíduo numa atividade. Para Weinberg e Gould (2001), isto pode ocorrer em virtude das pessoas buscarem adversários com diferentes níveis de habilidades ou para alcançar a superação de seus próprios limites. A Estética é considerada um dos motivos, pois, as pessoas utilizam a atividade física ou esportiva como forma de obter (conquistar) ou manter um corpo que seja considerado atraente e aprovado pela sociedade ou grupo em que o indivíduo está inserido. E por fim, o Prazer, motivo que está relacionado aos níveis intrínsecos de motivação, à sensação de bem-estar, de diversão e satisfação que a prática regular da atividade física e esportiva pode proporcionar (DECI; RYAN, 1985).

Considerando a importância de conhecer os motivos que estão associados à prática regular de atividades físicas e esportivas, este estudo tem por objetivo apresentar os principais motivos que levam atletas de Taekwondo a praticar regularmente esse esporte nas academias e clubes da cidade de Porto Alegre. 


\section{Método}

Procedimentos, Sujeitos e Instrumentos

Após a aprovação do Comitê de Ética em Pesquisa da Universidade Federal do Rio Grande do Sul sob o número 2007721, conforme previsto no projeto, os responsáveis pelos atletas foram contatados nos próprios locais de treinamento de Taekwondo. Todas as instituições visitadas - localizadas na cidade de Porto Alegre - eram filiadas a Confederação Brasileira de Taekwondo. Todos os locais de treinamento de Taekwondo contatados aceitaram participar deste estudo.

Os praticantes de Taekwondo foram convidados a responder um questionário referente à motivação. Sublinhava-se que eles eram livres de não participar ou de desistirem da pesquisa a qualquer momento, presente ou futuro, se assim o desejassem. Com a concordância verbal acordada, pedia-se que os praticantes de Taekwondo (os maiores de 18 anos) ou o (a) instrutor (a) assinassem (no papel de responsável) o Termo de Consentimento Livre e Esclarecido (TCLE). Por fim, considerando que não era exigida a identificação por nome dos participantes, os participantes estavam assegurados da confidencialidade de suas respostas. O inventário foi respondido individualmente e aplicado coletivamente a todos os praticantes de Taekwondo nos locais de treinamento.

Sendo assim, a amostra foi escolhida de acordo com a disponibilidade e a acessibilidade - amostra não-aleatória - conforme referem Maguire e Rogers (1989) e Thomas e Nelson (2002). Os 50 praticantes de Taekwondo, de ambos os sexos, com idades variando de 12 a 51 anos, implicados nesta pesquisa, foram recrutados em várias academias e clubes da cidade de Porto Alegre-RS.

Os participantes responderam ao Inventário de Motivação à Prática Regular de Atividade Física ou Esportiva (IMPRAFE-54) (BALBINOTTI; BARBOSA, 2006). Trata-se de um instrumento que avalia seis dimensões motivacionais: Controle de estresse, Saúde, Sociabilidade, Competitividade, Estética e Prazer. O IMPRAFE-54 é composto por 54 itens agrupados 6 a 6 , seguindo a sequência das dimensões a serem estudadas, a saber: controle de estresse (ex.: liberar tensões mentais), saúde (ex.: manter a forma física), sociabilidade (ex.: estar com amigos), competitividade (ex.: vencer competições), estética (ex.: manter bom aspecto) e prazer (ex.: meu próprio prazer). As respostas 
aos itens do IMPRAFE-54 são dadas conforme uma escala tipo Likert, bidirecional graduada em 5 pontos, indo de "isto me motiva pouquíssimo" (1) a "isto me motiva muitíssimo" (5). Cada dimensão é analisada e o resultado total também é obtido, pois todas as dimensões têm o mesmo número de questões. A validade e fidedignidade deste inventário foram testadas e demonstradas nos estudos de Barbosa (2005) e Balbinotti; Barbosa (2006).

\section{Apresentação e discussão dos resultados}

Os resultados serão apresentados através de uma análise descritiva, tendência central (média, media aparada 5\%, moda, mediana), dispersão (desvio padrão), distribuição (normalidade, assimetria e achatamento) dos dados obtidos com o instrumento utilizado nesta pesquisa (IMPRAFE-54). Para tanto, foi utilizado o programa estatístico SPSS 15.0.

Estatísticas descritivas gerais

Como se pode observar na Tabela 1, os índices obtidos relativos ao IMPRAFE-54, independente da variável controlada, variaram bastante, em valores nominais, $(22,96$ a 32,92$)$. O desvio-padrão seguiu nesta mesma ordem, variando de 0,96 a 1,31 .

As estatísticas de tendência central (mediana e média aparada a $5 \%$ ) de forma geral apresentaram valores próximos aos valores das médias. Quanto às modas, apenas as dimensões Controle de Estresse e Saúde não apresentaram múltiplas moda. As dimensões Saúde e Competitividade apresentaram a moda um pouco distante do valor da média. Os valores mínimo e máximo ficaram entre 8 e 40, independentemente da variável analisada. A relativa ausência de discrepâncias entre os resultados relativos às estatísticas de tendência central é um indício de que estas distribuições tendem à normalidade (o que será ocasionalmente testado). Estes resultados positivos relativos às estatísticas de tendência central são complementados por aqueles relativos às estatísticas de dispersão. 
Tabela 1: Estatísticas descritivas das dimensões do IMPRAFE-54

\begin{tabular}{|c|c|c|c|c|c|c|c|c|c|c|}
\hline \multirow[b]{2}{*}{ Dimensões } & \multicolumn{5}{|c|}{ Tendência Central e Não Central } & \multicolumn{3}{|c|}{ Normalidade } & \multirow{2}{*}{$\begin{array}{c}\text { Assimetria } \\
\text { Skewness/EPs }\end{array}$} & \multirow{2}{*}{$\begin{array}{l}\text { Achatamento } \\
\text { Kurtosis/EP }\end{array}$} \\
\hline & $\chi_{(\mathrm{DP})}$ & $\begin{array}{c}\text { Mínimo } \\
/ \\
\text { Máximo }\end{array}$ & $\mathbf{M}_{\mathrm{ed}}$ & $\begin{array}{c}\text { Trimed } \\
\mathbf{5} \%\end{array}$ & $\mathbf{M}_{\mathrm{od}}$ & S-W & GI & Sig & & \\
\hline $\begin{array}{l}\text { Controle de } \\
\text { Estresse }\end{array}$ & $22,96_{(1,31)}$ & $8-39$ & 23 & 22,93 & 23 & 0,102 & 50 & $0,200^{*}$ & $-0,00$ & $-1,76$ \\
\hline Saúde & $32,92(0,96)$ & $10-40$ & 35,5 & 33,53 & 40 & 0,174 & 50 & 0,001 & $-3,69$ & $-2,27$ \\
\hline Sociabilidade & $23,58_{(1,14)}$ & $11-40$ & 26,5 & 25,91 & $25^{a}$ & 0,090 & 50 & $0,200^{*}$ & $-0,53$ & $-1,22$ \\
\hline Competitividade & $23,06(1,28)$ & $8-40$ & 22 & 22,98 & $16^{\mathrm{b}}$ & 0,111 & 50 & 0,166 & 0,45 & $-1,58$ \\
\hline Estética & $25,96(1,11)$ & $8-40$ & 27 & 26,01 & 26 & 0,104 & 50 & $0,200^{*}$ & $-0,54$ & $-1,01$ \\
\hline Prazer & 32,40 (0.98) & $16-40$ & 35 & 32,85 & $35^{\mathrm{d}}$ & 0,233 & 50 & 0,000 & $-3,21$ & 0,27 \\
\hline
\end{tabular}

Múltiplas Modas: ${ }^{\mathrm{a}}(32,33) ;{ }^{\mathrm{b}}(21) ;{ }^{\mathrm{c}}(27) ;{ }^{\mathrm{d}}(40)$.

Quanto às estatísticas de distribuição, a aderência à normalidade foi testada com o auxílio do teste Shapiro-Wilk $(\mathrm{p}>0,05)$. Os resultados indicam que 4 das 6 dimensões aderem à normalidade (Controle de Estresse, Sociabilidade, Competitividade e Estética) (PESTANA; GAGEIRO, 2005).

Considerando os valores nominais, pode-se observar que a dimensão motivacional Saúde $(32,92)$ é a que mais motiva os praticantes de Taekwondo, seguida respectivamente pelas dimensões Prazer $(32,40)$, Estética $(25,96)$, Sociabilidade $(23,58)$, Competitividade $(23,06)$ e Controle de Estresse $(22,96)$.

A comparação entre os escores das dimensões motivacionais foi testada através do teste $t$ pareado para identificação de possíveis diferenças estatisticamente significativas $(p<0,05)$.

Comparações das médias

$\mathrm{Na}$ comparação das médias entre as dimensões motivacionais dos praticantes de Taekwondo foram utilizados testes paramétricos. Apesar da não aderência à normalidade de duas dimensões motivacionais (Saúde e Prazer), justifica-se a utilização de tais testes com base no Teorema do Limite Central (PESTANA; GAGEIRO, 2005), que preconiza a utilização dos referidos testes em amostras com número superior a 30 sujeitos.

A Tabela 2 mostra através do teste t pareado que não existem diferenças estatisticamente significativas $(p>0,05)$ entre as dimensões Pensar a Prática, Goiânia, v. 17, n. 1, p. 01-294, jan./mar. 2014 
Controle de Estresse e Competitividade; Controle de Estresse e Estética; Saúde e Prazer; Sociabilidade e Estética e Competitividade e Estética. Com isto, as dimensões que mais motivam os praticantes de Taekwondo são a Saúde $(X=32,92)$ e o Prazer $(X=32,40)$ estatisticamente indissociáveis $(\mathrm{p}>0,05)$, seguidos respectivamente, por um grupo de dimensões também estatisticamente indissociáveis $(\mathrm{p}>$ $0,05)$ : Estética $(X=25,96)$, Sociabilidade $(X=23,58)$, Competitividade $(X=23,06)$ e Controle de Estresse $(X=22,96)$ e, por último, um par de dimensões também estatisticamente indissociáveis Competitividade $(X=23,06)$ e Controle e Estresse $(X=22,96)$.

Tabela 2: Comparações entre os escores das dimensões motivacionais dos praticantes de Taekwondo

\begin{tabular}{lccc}
\hline Dimensões Pareadas & t & gl & p \\
\hline Controle de Estresse - Saúde & $-8,369$ & 49 &, 000 \\
Controle de Estresse - Sociabilidade & $-2,226$ & 49 &, 031 \\
Controle de Estresse - Competitividade & $-0,053$ & 49 &, 958 \\
Controle de Estresse - Estética & $-1,990$ & 49 &, 052 \\
Controle de Estresse - Prazer & $-7,099$ & 49 &, 000 \\
Saúde - Sociabilidade & 4,980 & 49 &, 000 \\
Saúde - Competitividade & 5,476 & 49 &, 000 \\
Saúde - Estética & 6,921 & 49 &, 000 \\
Saúde - Prazer & $-0,477$ & 49 &, 635 \\
Sociabilidade - Competitividade & 2,037 & 49 &, 047 \\
Sociabilidade - Estética & $-0,055$ & 49 &, 956 \\
Sociabilidade - Prazer & $-5,260$ & 49 &, 000 \\
Competitividade - Estética & $-1,883$ & 49 &, 066 \\
Competitividade - Prazer & $-6,131$ & 49 &, 000 \\
Estética - Prazer & -4.859 & 49 &, 000 \\
\hline
\end{tabular}

As dimensões Saúde e Prazer não surpreendem por serem aquelas que mais motivam os praticantes de Taekwondo, tendo em vista que a modalidade zela pelo bem-estar físico e mental de seus praticantes e cultiva valores pertinentes ao desenvolvimento da autonomia, sendo então sinais de motivação intrínseca (DECI, RYAN, 2000). Para Pergher; Klering; Balbinotti (2011), a dimensão motivacional Saúde costuma ser motivada extrinsecamente no período da adolescência, 
sendo seu valor determinado por fontes externas, com a possibilidade de ser internalizado com o decorrer do tempo, tornando-se então parte de um comportamento mais autônomo. A divulgação nos últimos tempos através dos diversos meios de comunicação acerca da importância e dos meios de se obter saúde talvez tenha influenciado na construção e internalização dessa instância. O Taekwondo, além de ser um exercício aeróbico intenso, desenvolve a flexibilidade, força, agilidade, potência, equilíbrio, noção espaço-temporal, entre outros (COSTA, 2010; MELHIM, 2001). Estas idéias estão de acordo com as concepções de Deci e Ryan (2000), para quem a relevância da Saúde decorre da sua íntima relação com a melhora da condição física e da formação de uma mente sadia.

Quanto à dimensão Prazer, Pergher; Klering; Balbinotti (2011), acredita que essa dimensão estar associada á prática regular de atividades físicas e esportivas se justifica em função das pessoas sentirem prazer em praticar aquilo que gostam e que sabem, pois o prazer provém das sensações de competência e de autonomia na realização da atividade, sendo símbolo do comportamento intrinsecamente motivado (DECI; RYAN, 2000). Juchem et al., (2007), afirmam que a responsabilidade e o desempenho em busca de um objetivo são, juntamente com o prazer, fatores que auxiliam na permanência da atividade. Ainda, Saldanha et al. (2008), realizaram um estudo com adolescentes do sexo feminino sobre motivação relacionada ao prazer e tiveram como resultado elevados índices de motivação intrínseca durante toda a adolescência. Portanto, o prazer pode ser considerado um motivo primordial para a prática regular dos esportes.

A constatação de que as dimensões Saúde e Prazer são as que mais motivam os praticantes de Taekwondo pode estar relacionada à satisfação das necessidades psicológicas destes praticantes, tendo em vista os resultados positivos que a prática dessa modalidade poderá trazer em médio e longo prazo (DECI; RYAN, 2000).

As dimensões, Estética, Sociabilidade, Competitividade e Controle de Estresse formaram um grupo que não apresentam diferenças estatisticamente significativas entre si. Esse resultado causa certa surpresa, pois se acreditava que a Sociabilidade e a Competitividade fossem um dos motivos de forte presença nesta modalidade, já que o Taekwondo propicia a socialização e a integração do grupo, valorizando o respeito, a colaboração e o auxílio entre os colegas. Além disso, percebe-se muita união entre seus membros nas situações de competição, especi- 
almente após o reconhecimento da modalidade como esporte olímpico (RIOS, 2005). O Taekwondo é uma modalidade esportiva que se configura enquanto formadora da cidadania, através do cultivo de valores - disciplina, respeito, perseverança, integridade, cortesia e autocontrole -, que são instâncias fundamentais para o desenvolvimento do bom convívio social e do crescimento pessoal.

Outra constatação importante foi a de que a dimensão Estética motiva os praticantes de Taekwondo da mesma forma que a Sociabilidade, Competitividade e Controle de Estresse. Esse resultado pode estar relacionado à exigência no sentido da estética das técnicas para as quais os aprendizes demandam muito esforço com o intuito de alcançar seus objetivos. Pergher; Klering; Balbinotti (2011) afirma que os jovens se comparam muito entre si com relação à aparência física e, por isso, a estética tem um importante papel na motivação para a prática esportiva, além de sua importância na sociedade ocidental como um todo, culminando na busca constante por um corpo "aceitável". O estudo de Barbosa et al. (2007), com adolescentes do sexo masculino, indicou a presença de altos índices de motivação relacionada à Estética durante todo este período da vida, havendo inclusive um aumento nesses valores no final da adolescência.

Quanto à dimensão motivacional Competitividade, este estudo mostra que os taekwondistas não se sentem muito motivados pela competição. De acordo com De Rose Jr.; Tricoli (2005), competir significa estar preparado para enfrentar os desafios da demanda situacional na busca do desempenho no mais alto grau de excelência à atividade e não medindo esforços para atingir os melhores resultados. Para Pergher; Klering; Balbinotti (2011), diversas vezes o momento da competição é onde essa situação ocorre; dificilmente nos treinamentos. É provável que os praticantes de Taekwondo não sejam motivados por esta dimensão em função de que nas aulas é comum esforçar-se ao máximo, não deixando o sentimento de superação e de vitória para os torneios; mas sim para os diversos momentos da aula em que ocorrem pequenas competições a título de aprimorar as técnicas de chute, defesa e socos. Nos treinos, apesar de geralmente ocorrerem combates livres ou combates combinados, não é comum a nomeação de vencedores. Normalmente, os treinamentos se caracterizam pela correção sistemática das técnicas de execução dos movimentos.

Pensar a Prática, Goiânia, v. 17, n. 1, p. 01-294, jan./mar. 2014 
A dimensão Controle de Estresse apresenta valores mais altos na população adulta, configurando-se a atividade física como uma das maneiras de aliviar o estresse (PERGHER; KLERING; BALBINOTTI, 2011; GOYEN; ANSHEL,1998). Neste estudo, a dimensão Controle de Estresse é a que menos motiva os praticantes de Taekwondo. Talvez, porque durante as aulas se busca evitar o conflito com o adversário, procurando sempre agir em prol da defesa pessoal. Em outras palavras, a filosofia da arte marcial ensina o autocontrole e a cortesia. Isso pode culminar em comportamentos que afastam o indivíduo bem treinado das situações de estresse, bem como permite que ele atue de forma mais amena quando estas situações estressantes forem inevitáveis.

\section{Conclusão}

Este trabalho permitiu explorar possíveis diferenças e/ou semelhanças estatisticamente significativas encontradas nas respostas de 50 praticantes de Taekwondo de ambos os sexos, com idades variando de 12 a 51 anos, oriundos de academias e clubes da cidade de Porto Alegre-RS. A partir dos cuidados quanto aos procedimentos éticos e metodológicos, os resultados não indicam diferenças significativas $(\mathrm{p}<$ $0,05)$ nas médias das dimensões Controle de Estresse e Competitividade; Controle de Estresse e Estética; Saúde e Prazer; Sociabilidade e Estética e Competitividade e Estética. Mesmo que não seja recomendável generalizar os resultados obtidos nesta pesquisa, tendo em vista que o número de sujeitos não é suficiente para tal, é possível chegar a algumas conclusões importantes.

As dimensões que mais motivam os praticantes de Taekwondo são: a Saúde e o Prazer, estatisticamente indissociáveis $(\mathrm{p}>0,05)$. Estes resultados indicam que o reconhecimento dos benefícios que a prática do Taekwondo pode trazer a saúde e a participação em um esporte que traga prazer, satisfação pessoal ao praticante, pode fazer com que os taekwondistas permaneçam por mais tempo e mais motivados nas atividades propostas.

Finaliza-se este estudo indicando que estas conclusões podem ser úteis, particularmente para instrutores, professores e mestres, que buscam melhorar a qualidade de suas aulas e a formação de seus alunos. Aprender uma arte marcial abrange muito mais que a compreensão das normas e execução eficaz das técnicas. É fundamental a constru- 
ção de valores morais e éticos e a internalização da modalidade como modo de vida saudável.

Novos estudos devem ser conduzidos, a fim de se verificar a existência de possíveis diferenças estatisticamente significativas nas dimensões motivacionais de praticantes de Taekwondo, principalmente, com relação à faixa etária, o sexo, as categorias, os participantes de competição, o tempo de prática, entre outras, possibilitando assim a produção de conhecimentos mais específicos.

\title{
MOTIVATION TO PRACTICE REGULAR PHYSICAL ACTIVITY: A STUDY WITH PRACTITIONERS OF TAEKWONDO
}

\begin{abstract}
The aim of this study is to investigate, among the main motivational dimensions (Stress Control, Health, sociability, competitiveness, and Aesthetic Pleasure), which further motivates the regular practice of Taekwondo. The instrument used for data collection was Motivation Inventory Regulate the Practice of Physical Activities and / or Sports - IMPRAFE-54. The sample was composed of 50 practitioners of Taekwondo in the age group 12-51 years, of both sexes. Among the results showed that the motivational dimensions Health and Pleasure inseparable statistically $(\mathrm{p}<0.05)$ are the ones that motivate practitioners of Taekwondo.
\end{abstract}

Keywords: Motivation. Motor Activity. Sports.

\section{MOTIVACIÓN PARA PRACTICAR UNA ACTIVIDAD FÍSICA REGULAR: UN STUDIO CON LOS PRACTICANTES DE TAEKWONDO}

\section{Resumen}

El objetivo de este estudio es investigar, entre las principales dimensiones de motivación (control del estrés, salud, placer sociabilidad, la competitividad y la estética), lo que más motiva a la práctica regular de Taekwondo. El instrumento utilizado para la recolección de datos es el Inventario de Motivación Reglamentar la Práctica de Actividades Físicas y / o Deportes - IMPRAFE-54. La muestra incluyó a 50 practicantes de Taekwondo en el grupo de edad 12-51 años, de ambos sexos. Entre los resultados mostraron que la motivación de la Salud y Placer dimensiones inseparables de vista estadístico $(p<0,05)$ son los que motivan a los practicantes de Taekwondo.

Palabras clave: Motivación. Actividad Motora. Deportes.

\section{Referências}

BALBINOTTI, M. A. A. Inventário de Motivação à Pratica Regular de Atividade Física (IMPRAFE-126). $2^{\mathrm{a}}$ versão. Laboratório 
de Psicologia do Esporte - Universidade Federal do Rio Grande do Sul: Porto Alegre, 2004.

- Inventário de Motivos para a Prática Regular de Atividades Físicas e Esportivas (IMPRAFE-132). 3. versão. Montréal, QC: Service d'Intervention et de Recherche en Orientation et Psychologie, SIROP, 2010.

LEITE GONÇALVES, R.; BALBINOTTI, M. A. A. IMPRAFE-132: Um novo instrumento de avaliação de motivos à prática regular de atividade física e esportiva. In: VII CONGRESSO INTERNACIONAL DE EDUCAÇÃO FÍSICA E MOTRICIDADE HUMANA E XIII SIMPÓSIO PAULISTA DE EDUCAÇÃO FÍSICA, Anais, Rio Claro - SP, 2011.

BALBINOTTI, M. A. A.; BARBOSA, M. L. L. Inventário de Motivação à Prática Regular de Atividade Física (IMPRAFE-54). Laboratório de Psicologia do Esporte - UFRGS. Porto Alegre, 2006.

. Análise da consistência interna e fatorial confirmatória do IMPRAFE-126 com praticantes de atividades físicas gaúchos. PsicoUSF, São Paulo, v. 13, n. 1, p.1-12, 2008.

BALBINOTTI, M. A. A.; CAPOZZOLI, C. J. Motivação à prática regular de atividade física: Um estudo exploratório com praticantes em academias de ginástica. Revista Brasileira de Educação Física e Esporte, v. 22, n. 1, p. 63-80, 2008.

BALBINOTTI, M. A. A.; SALDANHA, R. P.; BALBINOTTI, C. A. A. Dimensões motivacionais de basquetebolistas infanto-juvenis: Um estudo segundo o sexo. Motriz, Rio Claro, v. 15, n. 2, p. 318-329, 2009.

Balbinotti, M. A. A.; Silva, M. Inventário de Motivação Aplicado a Atividade Física (IMPRAFE-126). Laboratório de Psicologia do Esporte. Universidade Federal do Rio Grande do Sul: Porto Alegre, 2003.

BARBOSA, M. L. L. Propriedades métricas do inventário de motivação à prática regular de atividade física (IMPRAF-126). 140 p. Dissertação (Mestrado em Ciências do Movimento Humano) - 
Escola de Educação Física, Universidade Federal do Rio Grande do Sul, Porto Alegre, 2005.

BARBOSA, M. L. L. et al. A motivação à prática de atividade física regular relacionada à estética em adolescentes do sexo masculino. Coleção Pesquisa em Educação Física, v.6, n.2, 2007.

CAPOZZOLI, C. J. Motivação à prática de atividades físicas: um estudo com praticantes de academias de ginástica de Porto Alegre. 153 p. Dissertação (Mestrado em Ciências do Movimento Humano) Escola de Educação Física, Universidade Federal do Rio Grande do Sul, Porto Alegre, 2006.

COSTA, R. O conhecimento da história e da filosofia do Taekwondo dos taekwondistas santoamarenses. 80 p. Monografia (Graduação em Educação Física) - Curso de Educação Física, Universidade Federal de Santa Catarina, Florianópolis, 2010.

DE ROSE JR, D.; TRICOLI, V. Basquetebol: conceitos e abordagens gerais. In: DE ROSE JR., D.; TRICOLI, V. (orgs.) Basquetebol: uma visão integrada entre ciência e prática. Barueri, SP: Manole, p. 1-14, 2005.

DECI, E. L.; RYAN, R. M. Intrinsic Motivation and selfdetermination in human behavior. New York: Plenum, 1985.

. The "what" and "why" of goal pursuits: human needs and the self-determination of behavior. Psychological Inquiry, v.11, n.4, p. 227-268, 2000.

FONTANA, P. S. A motivação na ginástica rítmica: um estudo descritivo correlacional entre as dimensões motivacionais e a autodeterminação em atletas de 13 a 16 anos. 192 p. Dissertação (Mestrado em Ciências do Movimento Humano) -Programa de PósGraduação em Ciências do Movimento Humano, Escola de Educação Física, Universidade Federal do Rio Grande do Sul, Porto Alegre, 2010.

GOYEN, M. J.; ANSHEL, M. H. Souse of acute competitive stress and use of coping strategies as a function of age and gender. Journal of Applied Developmental Psychology, v. 3, n. 19 p. 469-486, 1998. 
JUCHEM, L. et al. A motivação para a prática regular de atividades físicas: um estudo descritivo-exploratório com tenistas do sexo masculino de 13 à 16 anos. Coleção Pesquisa em Educação Física, vol. 6, n. 2, p. 19-24, 2007.

McDONALD, D.; HODGOON, J. Psychological effects of aerobic fitness training. New York: Springer-Verlag, 1991.

MAGUIRE, T.; ROGERS W. Proposed solutions for non randomness in educational research. Canadian Journal of Education, v. 14, n. 2, p. 170-181, 1989.

MELHIM, A. F. Aerobic and anaerobic power responses to the practice of taekwondo. Revista Brasileira de Medicina do Esporte, São Paulo, v. 35, p. 231-234, ago., 2001.

PERGHER, T. K.; KLERING, R. T.; BALBINOTTI, C. A. A. Motivação à prática regular de atividades esportivas: um estudo com praticantes de basquetebol escolar (13 a 16 anos). Lecturas Educación Física y Deportes (Buenos Aires), v. 15, p. 1/153-1, 2011.

PESTANA, M. H.; GAGEIRO, J. G. Análise de dados para ciências sociais: a complementaridade do SPSS. 4. ed. Lisboa: Edições Silabo, 2005.

RIOS, G. B. O Processo de esportivização do Taekwondo. Pensar a Prática, Goiânia, v.8, n.1, p. 37-54, jan./jun., 2005.

SALDANHA, R. P. et al. A motivação à prática de atividade física regular relacionada ao prazer em adolescentes do sexo feminino. Coleção Pesquisa em Educação Física, v.7, n.1, 2008.

SILVA, A. M. A. C. da. Reconectando a sociabilidade on-line e offline: trajetórias, formação de grupos e poder em canais geográficos no Internet Relay Chat (IRC). 212 p. 2000. Dissertação (Mestrado em Sociologia)-Instituto de Filosofia e Ciências Humanas, Universidade Estadual de Campinas, Campinas, 2000.

THOMAS, J.; NELSON, J. Métodos de Pesquisa em Atividades Físicas. 3. ed. Porto Alegre: Artmed, 2002. 
WEINBERG, R.; GOULD, D. Fundamentos da Psicologia do Desporto e do Exercício. Porto Alegre: Artmed, 2001. 560 p.

Recebido em: 28/02/2012

Revisado em: 28/07/2012

Aprovado em: 05/02/2013

Endereço para correspondência

lisianerocha@hotmail.com

Lisiane Borges Rocha Sampedro

Escola de Educação Física da Universidade Federal do Rio Grande do Sul

Rua Felizardo, $n^{\circ} 750$ - Jardim Botânico

Porto Alegre / RS 